\title{
RAL GTPases mediate multiple myeloma cell survival and are activated independently of oncogenic RAS
}

Haematologica 2020

Volume 105(9):2316-2326

\section{Correspondence: \\ TORSTEN STEINBRUNN \\ steinbrunn_t@ukw.de}

Received: March 26, 2019.

Accepted: October 10, 2019.

Pre-published: October 10, 2019.

doi:10.3324/haematol.2019.223024

(C)2020 Ferrata Storti Foundation

Material published in Haematologica is covered by copyright. All rights are reserved to the Ferrata Storti Foundation. Use of published material is allowed under the following terms and conditions:

https://creativecommons.org/licenses/by-nc/4.0/legalcode. Copies of published material are allowed for personal or internal use. Sharing published material for non-commercial purposes is subject to the following conditions:

https://creativecommons.org/licenses/by-nc/4.0/legalcode, sect. 3. Reproducing and sharing published material for commercial purposes is not allowed without permission in writing from the publisher.

\author{
Marcel Seibold, ${ }^{1}$ Thorsten Stühmer, ${ }^{2}$ Nadine Kremer, ${ }^{2}$ Anja Mottok, ${ }^{3}$ \\ Claus-Jürgen Scholz, ${ }^{4}$ Andreas Schlosser, ${ }^{5}$ Ellen Leich, ${ }^{6}$ Ulrike Holzgrabe, ${ }^{7}$ \\ Daniela Brünnert, ${ }^{2}$ Santiago Barrio, ${ }^{8}$ K. Martin Kortüm, ${ }^{1}$ \\ Antonio G. Solimando, ${ }^{1}$ Manik Chatterjee, ${ }^{2}$ Hermann Einsele, ${ }^{1}$ \\ Andreas Rosenwald, ${ }^{6}$ Ralf C. Bargou ${ }^{2}$ and Torsten Steinbrunn ${ }^{1}$
}

${ }^{1}$ Department of Medicine II, University Hospital of Würzburg, Würzburg, Germany; ${ }^{2}$ Comprehensive Cancer Center Mainfranken, Chair of Translational Oncology, University Hospital of Würzburg, Würzburg, Germany; ${ }^{3}$ nstitute of Human Genetics, University of Ulm, Ulm, Germany; ${ }^{4}$ Core Unit Systems Medicine, University of Würzburg, Würzburg, Germany; ${ }^{5}$ Rudolf Virchow Center for Experimental Biomedicine, University of Würzburg, Würzburg, Germany; 'Institute of Pathology, University of Würzburg, Würzburg, Germany; ${ }^{7}$ Institute of Pharmacy and Food Chemistry, University of Würzburg, Würzburg, Germany and ${ }^{8} \mathrm{Hematology}$ Department, Hospital 12 de Octubre, Complutense University, Madrid, Spain

\section{ABSTRACT}

$\longrightarrow$ ncogenic RAS provides crucial survival signaling for up to half of multiple myeloma (MM) cases, but has so far remained a clinically undruggable target. RAS-like protein (RAL) is a member of the RAS superfamily of small GTPases and is considered to be a potential mediator of oncogenic RAS signaling. In primary MM, we found RAL to be overexpressed in the vast majority of samples when compared with pre-malignant monoclonal gammopathy of undetermined significance or normal plasma cells. We analyzed the functional effects of RAL abrogation in myeloma cell lines and found that RAL is a critical mediator of survival. RNAi-mediated knockdown of RAL resulted in rapid induction of tumor cell death, an effect which was independent from signaling via mitogen-activated protein kinase, but appears to be partially dependent on Akt activity. Notably, RAL activation was not correlated with the presence of activating RAS mutations and remained unaffected by knockdown of oncogenic RAS. Furthermore, transcriptome analysis yielded distinct RNA expression signatures after knockdown of either RAS or RAL. Combining RAL depletion with clinically relevant anti-myeloma agents led to enhanced rates of cell death. Our data demonstrate that RAL promotes MM cell survival independently of oncogenic RAS and, thus, this pathway represents a potential therapeutic target in its own right.

\section{Introduction}

Mutated RAS is one the most frequent oncogenic drivers in human cancers, yet it has so far confounded efforts to render it a clinically exploitable drug target. ${ }^{1-4}$ Consequently, the identification and targeting of RAS effector pathways has been pursued to establish therapeutic approaches that counter RAS-driven tumors. ${ }^{5-7}$ Multiple myeloma (MM) harbors oncogenic NRAS or KRAS mutations in up to half of the cases and we have shown that RNA-mediated knockdown of oncogenic RAS induces apoptosis in $\mathrm{MM}$ cell lines. ${ }^{8-10}$

In vitro, the so-called classical RAS-associated pathways which signal via RAF/MAPK and PI3K/Akt, respectively, have been studied at different levels in MM cells and have been shown to be crucial for MM cell survival. ${ }^{11-19}$ In addition, we have demonstrated that although inhibition of one or both of these pathways can strongly affect MM cell growth and survival in vitro, ${ }^{8,14}$ their constitutive activation appears not to be directly correlated with the presence of oncogenic RAS.,11 However, early clinical trials including MM patients treated with pharmacological inhibitors of either one of these pathways have shown only limited efficacy, ${ }^{20-22}$ 
whereas combined blockade in patients with solid tumors resulted in high levels of toxicity. ${ }^{23-26}$ The identification of alternative RAS-driven pathways to target MM cells is therefore highly warranted.

Here, we investigated the functional role of RAS-like protein (RAL) in MM, which has sometimes been branded "the third pathway" in the context of RAS-dependent oncogenic signaling. ${ }^{27-29}$ RAL belongs to the RAS superfamily of small GTPases that - like RAS itself - are characterized by cycling between a GTP-bound active and a GDP-bound inactive state. The two isoforms of RAL: RALA and RALB have both been shown to be involved in malignant transformation, tumor cell survival, and tumor cell growth and metastasis, although their functional role(s) may depend to some extent on the tumor entity and/or model tested. ${ }^{30-32}$

In our study, we sought to analyze the functional importance of RAL in MM as bona fide downstream effector of oncogenic RAS by using RNAi-mediated knockdown approaches. We found that RAL is important for MM cell survival, but that its constitutive activation is not directly linked to oncogenic RAS. Furthermore, knockdown of RAL entails very different transcriptomic changes than RAS depletion. Therefore, we infer that the RAL pathway constitutes a potential clinical target in its own right.

\section{Methods}

\section{Culture of MM cell lines and preparation of primary MM cells}

Cell culture conditions of human myeloma cell line (HMCL) and isolation of CD138-positive primary MM cells were previously described. ${ }^{33}$ Bone marrow aspirates of $\mathrm{MM}$ patients were obtained after informed consent according to the Declaration of Helsinki, and with permission of the Ethics Committee of the University of Würzburg (reference no. 76/13). See the Online Supplementary Materials and Methods for details.

\section{Immunohistochemical stainings of bone marrow biopsies}

To evaluate protein expression of the RAL isoforms in plasma cells we performed immunohistochemical analysis in formalinfixed, paraffin-embedded bone marrow biopsies from 26 patients with $\mathrm{MM}$ as previously described., ${ }^{8,14}$ For comparison, we analyzed patients with monoclonal gammopathy of undetermined significance (MGUS) $(n=10)$ and bone marrow trephines containing reactive, polyclonal plasma cells $(n=5)$. Slides were evaluated by experienced hematopathologists. See the Online Supplementary Materials and Methods for details.

\section{Cell death assay}

Fractions of unaffected and (pre-)apoptotic cells were measured by flow cytometry after staining with propidium iodide (PI) and annexin V labeled with either PromoFluor 647, allophycocyanin (APC) or fluorescein isothiocyanate (FITC) as previously described. ${ }^{34}$ Cell death measurements were conducted at days 3 and 4 after transfection.

\section{Cell metabolism, proliferation and cell cycle assays}

Alamar Blue and bromodeoxyuridine (BrdU)/PI assays were performed to analyze cell metabolism, proliferation and cell cycle distribution after RAL knockdown or pharmacological inhibition with RBC8. See the Online Supplementary Materials and Methods for details.

\section{Construction of shRNA expression vectors}

Construction of pSUPER-based small hairpin RNA (shRNA) expression vectors was performed as previously described. ${ }^{35}$ See the Online Supplementary Materials and Methods for sequences. ${ }^{36,37}$

\section{Transfection of MM cells by electroporation}

Transient transfection of HMCL was previously described in detail. ${ }^{34}$ HMCL were electroporated with pSUPER-based shRNA expression vectors. ShRNA expression plasmid concentrations in the final electroporation mix were $20 \mu \mathrm{g} / \mathrm{mL}(15 \mu \mathrm{g} / \mathrm{mL}$ for transfections with subsequent drug treatment). Strongly transfected cells were purified by microbead selection for co-expressed CD4 or, in the case of AMO-1, by fluorescence-activated cell sorting for co-expressed enhanced green-fluorescent protein (EGFP).

\section{RALA activity assay}

INA- 6 and MM.1S cells were transfected with shRNA expression plasmids and harvested two days after electroporation. The activation status of RALA was measured using the RAL Activation Assay from Cell Biolabs (no. STA-408, San Diego, CA, USA) according to the manufacturer's instructions. Subsequent Western blotting was performed to analyze RAL-GTP levels and total RAL protein loads. Antibodies against RALA were diluted 1:500 or 1:1,000.

\section{Western analysis}

Western blotting of cell lysates was performed according to standard protocols as previously described. ${ }^{12,34}$ See the Online Supplementary Materials and Methods for details.

\section{RNA sequencing analysis}

For transcriptome analyses, MM.1S cells were transfected with pSUPER-based shRNA expression vectors against either KRAS or RALA. Control cells were transfected with empty pSUPER plasmids. RNA sequencing data are deposited in Gene Expression Omnibus in entry GSE126794. See the Online Supplementary Materials and Methods for details.

\section{Mass spectrometry-based interactome analysis}

To identify RAL interaction partners we performed quantitative mass spectrometric analysis of MM.1S cells with stable expression of HA-tagged RALA protein. Detailed description of sample preparation and analysis is provided in the Online Materials and Methods and by Cox et al. .8,39 $^{3}$

\section{Statistical analysis}

Statistical significance $(P<0.05)$ was determined by a two-tailed Student's $t$-test. Three independent experiments were performed.

\section{Results}

\section{RAL expression in multiple myeloma cells}

RAL protein expression in a panel of MM cell lines $(n=7)$ and primary MM samples ( $n=10)$ was analyzed for each isoform using Western blotting. Both proteins were detected at fairly equal levels in all (RALA), and in 6 of 7 (RALB) HMCL, respectively (Figure 1A, top). Cell line U266 was notable for its complete lack of RALB expression. Interestingly, all cell lines showed constitutive RALA activation through the presence of GTP-bound RALA as detected by a pulldown assay (Figure 1A, bottom). In CD138-positive primary MM cells isolated from bone marrow aspirates of $\mathrm{MM}$ patients, both RAL proteins were always present. Accounting for differences in the amounts of sample loading, RALA and RALB expression 
A

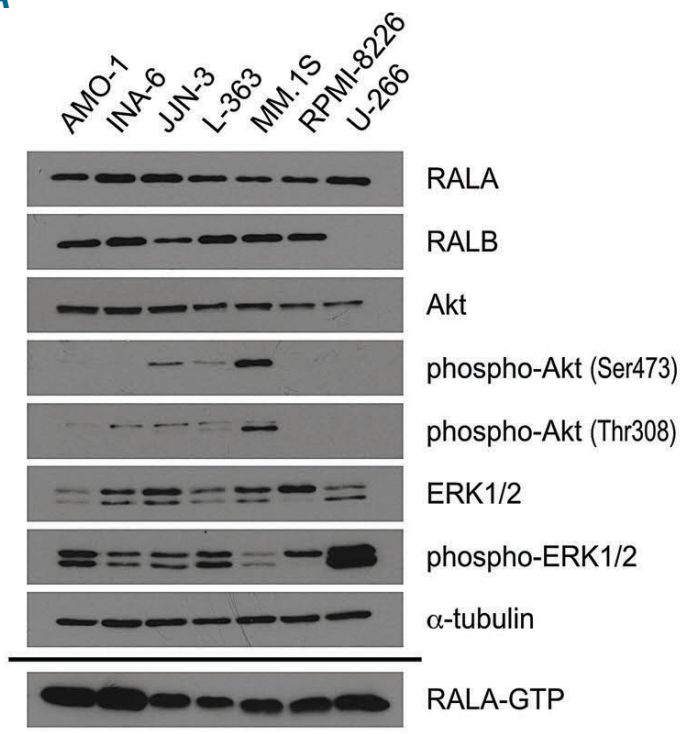

B

\begin{tabular}{|c|c|}
\hline$-\cdots--\infty-\cdots$ & RALA \\
\hline$-\cdots--\infty-\cdots$ & RALB \\
\hline$----\cdots-\cdots$ & Akt \\
\hline$-\quad---\infty$ & phospho-Akt (Ser473) \\
\hline$=-\quad-\infty$ & phospho-Akt (Thr308) \\
\hline$\Rightarrow \quad=-=-ー-\cdots$ & ERK1/2 \\
\hline$-x==$ & phospho-ERK1/2 \\
\hline-------1 & $\alpha$-tubulin \\
\hline
\end{tabular}

C

CD138
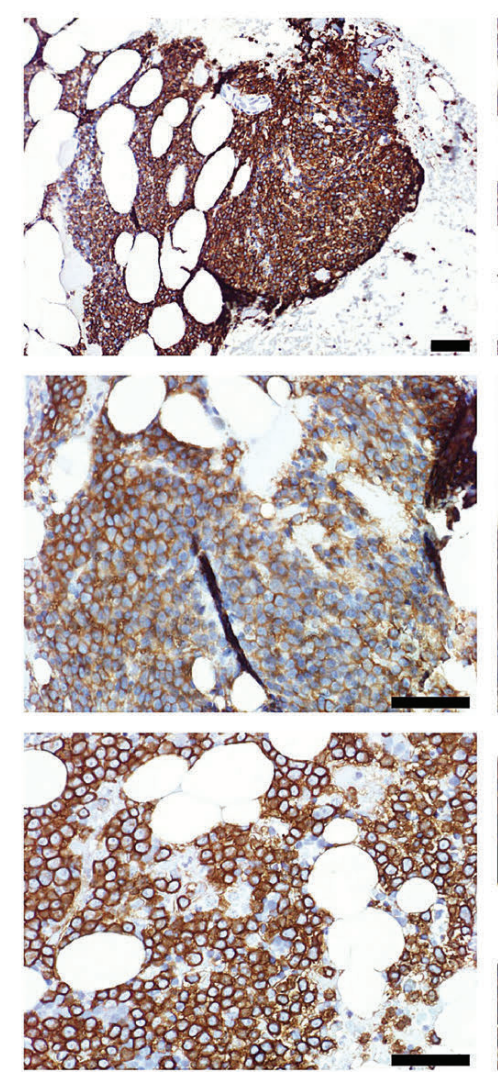

RALA
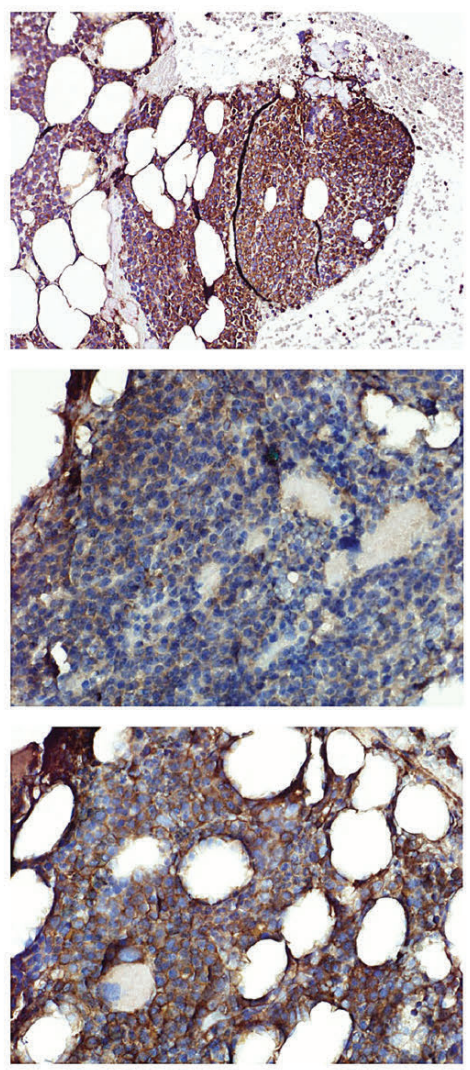

RALB
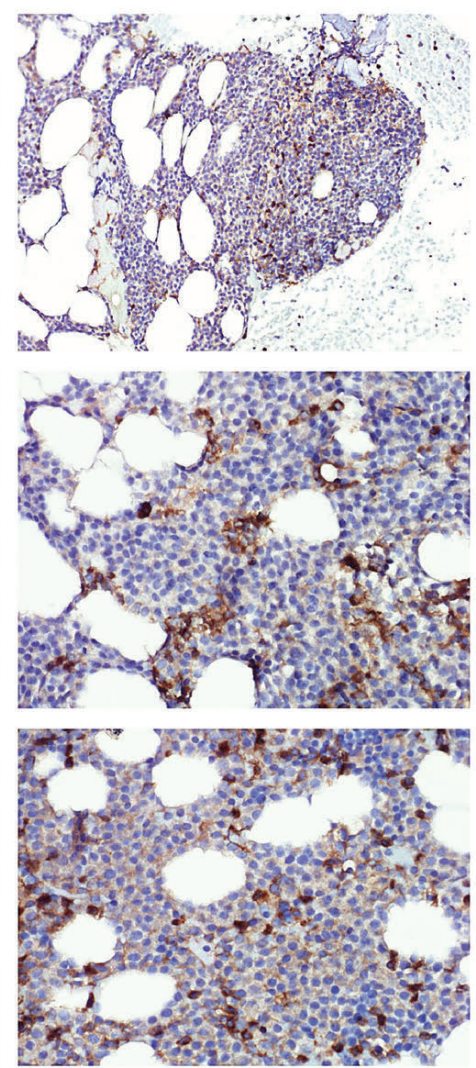

Figure 1. Expression of the two RAL isoforms in multiple myeloma cells. Representative Western analyses showing expression levels of RALA, RALA-GTP and RALB as well as expression and phosphorylation levels of MAPK and Akt signaling in (A) human myeloma cell line $(\mathrm{HMCL})(\mathrm{n}=7)$ and $(B)$ primary MM samples $(n=10)$. $\alpha-$ tubulin served as loading control. (C) In situ expression of RALA and RALB in bone marrow trephines of multiple myeloma (MM) patients ( $\mathrm{n}=26$ ). CD138 staining as well as RALA and RALB staining shown for 3 different patients (I, II, III) at 200x (I) and 400x (II, III) magnification. Scale bars: 50 um. Samples I, II, and III correspond to sample numbers 22,25 , and 26 in the Online Supplementary Table S2, respectively. 
A

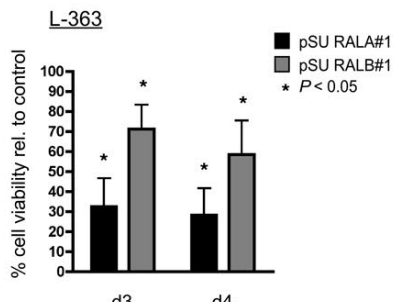

C

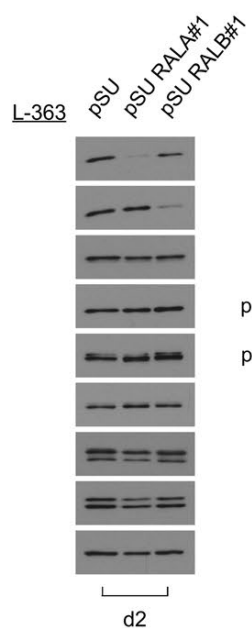

$\underline{\mathrm{L}-363}$

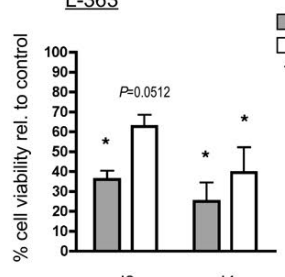

d3 $\quad$ d4
B

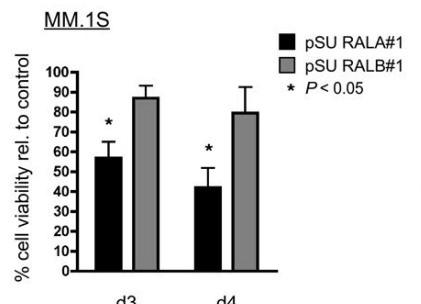

d3 d4

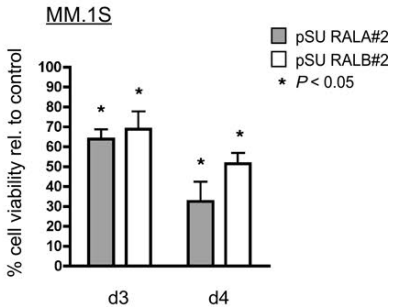

d3 $\quad$ d4

D
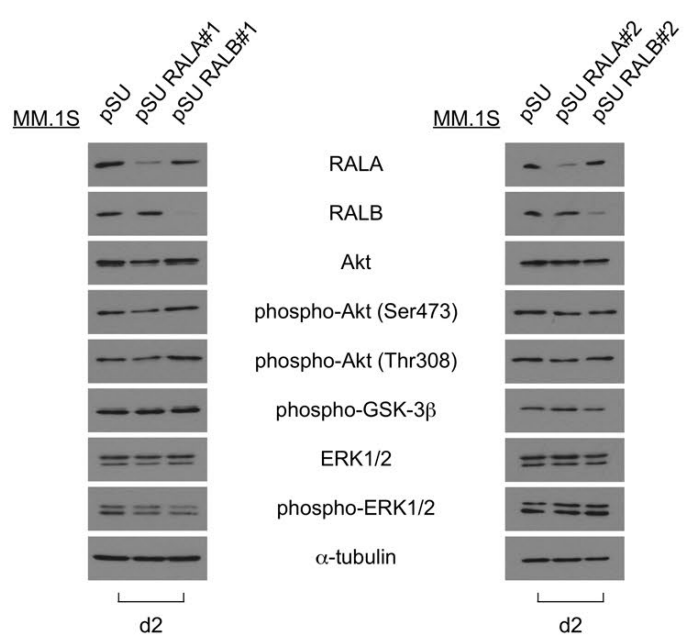

$\mathbf{F}$
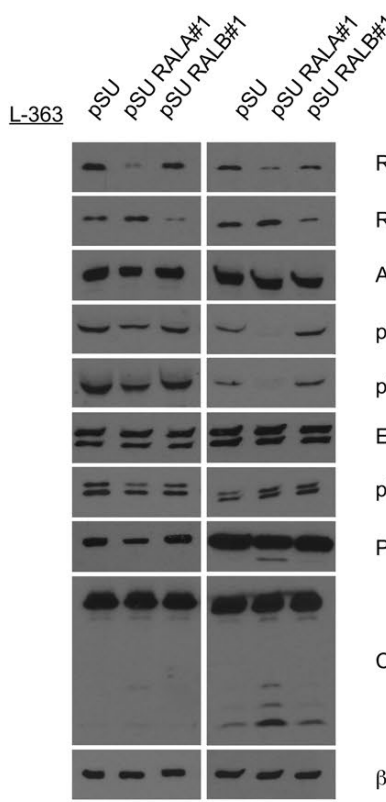

RALA

RALB

Akt

phospho-Akt (Ser473)

phospho-Akt (Thr308)

ERK1/2

phospho-ERK1/2

PARP-1

Caspase 3

$\beta$-actin
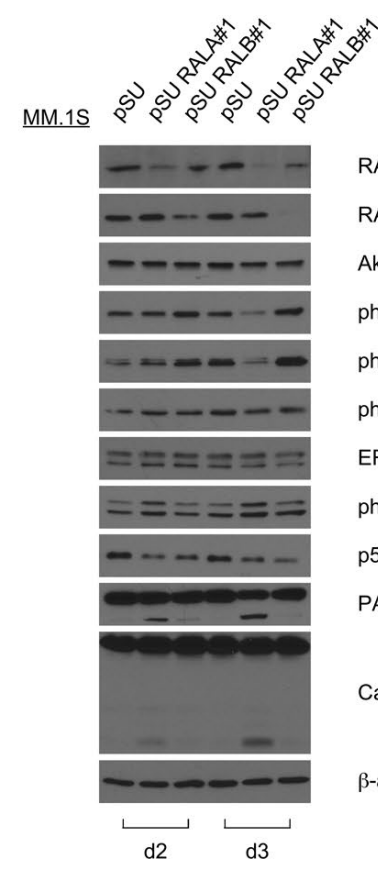

RALA

RALB

Akt

phospho-Akt (Ser473)

phospho-Akt (Thr308)

phospho-GSK-3 $\beta$

ERK1/2

phospho-ERK1/2

p53

PARP-1

Caspase 3

$\beta$-actin

d2 $\quad$ d3

Figure 2. Effects of abrogation of RAL signaling with different small hairpin RNA expression vectors on multiple myeloma cell survival and signaling. RALA and RALB knockdown was achieved with two different small hairpin RNA (shRNA) expression vector constructs for each RAL isoform in L-363 cells (A) and MM.1S cells (B). Upon RALA knockdown, MM cell survival was significantly reduced 3 days and 4 days after electroporation. Similarly, RALB knockdown also reduced cell survival, but to a lesser extent than RALA depletion. Shown are mean values and standard deviation (SD) from three independent experiments. Percentages were calculated relative to the respective empty vector control. Cell viability was monitored by annexin V/PI staining. Exemplarily, Western blots of L-363 (C) and MM.1S (D) cell lysates show that RAS-dependent signaling in form of ERK1/2 phosphorylation and PI3K-dependent signaling illustrated by Akt and GSK-3 $\beta$ phosphorylation are not influenced by RALA or RALB knockdown 2 days after electroporation. (E) L-363 and (F) MM.1S cells were transfected with shRNA targeting RALA or RALB and purified cells were harvested after 2 and 3 days. Onset of apoptosis after RALA knockdown as indicated by cleavage of PARP 1 and caspase-3 was accompanied by reduction of phosphorylated Akt after 3 days, whereas after 2 days, signaling remained still unchanged. $\alpha$-tubulin and $\beta$-actin served as loading control. 
A

INA-6

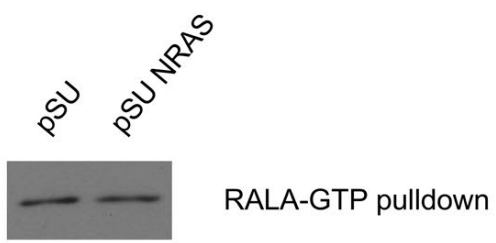

$\longrightarrow$ RALA total load

$\alpha-\alpha$-tubulin total load

- NRAS

- $\quad \alpha$-tubulin
MM.1S

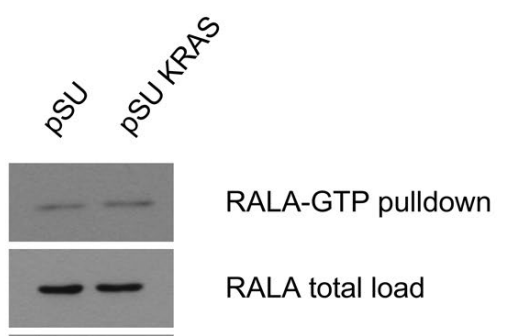

B

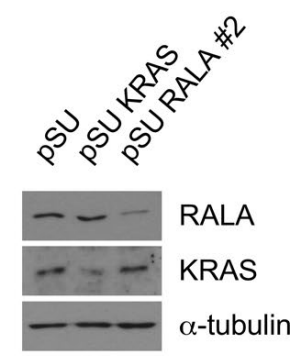

C

MM.1S

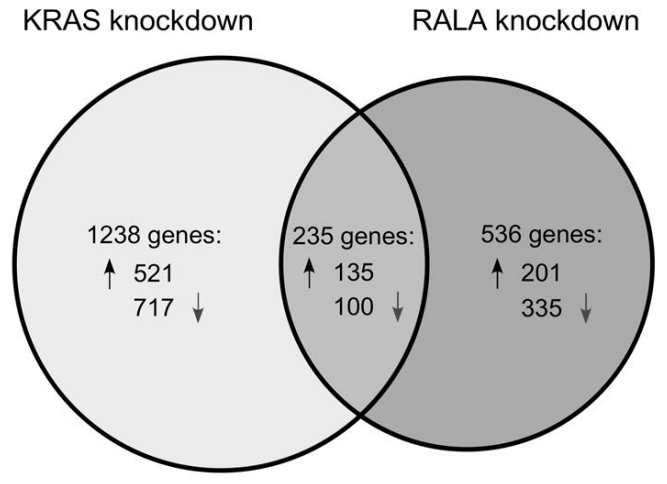

D $\alpha$-tubulin total load

KRAS

$\alpha$-tubulin

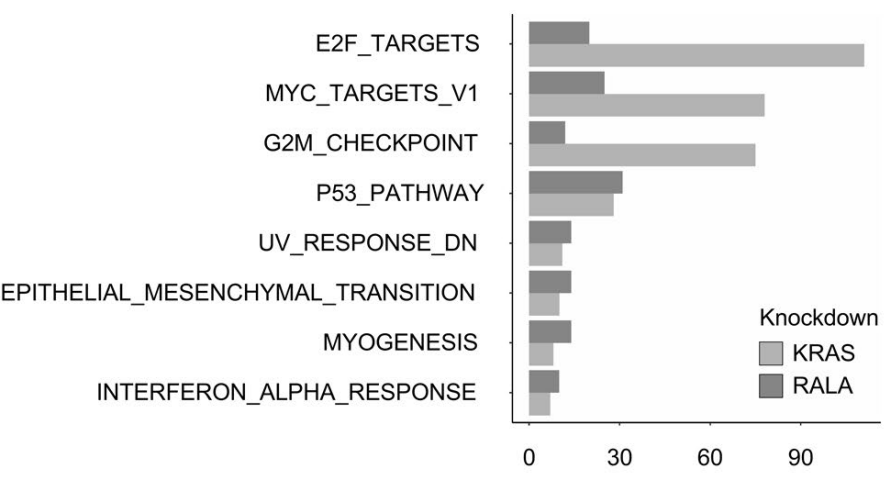

Differentially Expressed Genes

Figure 3. Distinct regulation of the RAL and RAS pathways. (A) NRAS (G12D)-mutated INA-6 cells were transfected with a samII hairpin RNA (shRNA) expression vector against mutated NRAS, KRAS (G12A)-mutated MM.1S cells were transfected with an shRNA expression vector against mutated KRAS. As shown by Western analyses, RALA activation was not changed by depletion of oncogenic RAS (and its cognate wild-type form) in either cell line. RALA activation was measured by RALA-GTP pulldown with RALPB1 protein-binding domain agarose beads 48 hours $(\mathrm{h})$ after transfection. RALA and $\alpha$-tubulin total load samples were taken before the pulldown procedure. (B) To analyze RAL- versus RAS-dependent gene expression, MM.1S cells were transfected with shRNA expression vectors against KRAS or RALA and successful knockdown was confirmed by Western blotting. RNA was isolated $48 \mathrm{~h}$ after electroporation and analyzed with RNA-Seq. Three independent experiments were performed. (C) Of 1,473 genes that were expressed differentially after KRAS knockdown, 656 were up- and 817 downregulated. After RALA knockdown, 771 genes showed an altered expression, whereof 336 were up- and 435 downregulated. Of the 235 genes in the overlap, 135 were up- and 100 downregulated under both conditions. The diagram shows all genes with altered expression with a false discovery rate (FDR) $<0.05$. In total, 28,440 genes were analyzed. (D) Ontology mapping of differential gene expression highlighting the most distinct functional gene groups with relevance for MM growth and survival after RAL versus RAS knockdown was performed using the Molecular Signatures Database Hallmark Gene Set Collection. ${ }^{40}$ Adjusted $P$-value $<0.05$.

again appeared quite similar between primary samples, with a few notable digressions to the upside (RALA in samples 1 and 5, RALB in sample 5; Figure 1B).

Expression of RAL isoforms in primary plasma cells was also analyzed in situ by immunohistochemical staining of bone marrow biopsies from MM patients $(n=26)$ and compared with sections from non-MM $(n=5)$ and MGUS patients $(n=10)$. Co-staining was performed with the plasma cell marker CD138 (Figure 1C). Normal plasma cells showed no detectable expression of RALA except for one sample, which displayed weak staining in $10 \%$ of the cells. All normal plasma cell samples were negative for
RALB staining. In 5 of 10 samples with pre-malignant MGUS plasma cells RALA was not detectable. The remaining 5 samples showed slightly elevated RALA expression levels. RALB expression did not reach the detection level in any MGUS sample (Online Supplementary Table $S 1$ and Online Supplementary Figure S1). In contrast, 20 of 26 primary samples from MM patients showed medium to strong RALA expression in $80 \%$ to $100 \%$ of the cells. Two samples showed RALA expression in $25 \%$ and $50 \%$ of the cells, respectively. In 14 of 26 samples, RALB stained weakly in at least $5 \%$ of MM cells (Online Supplementary Table S2). 
A

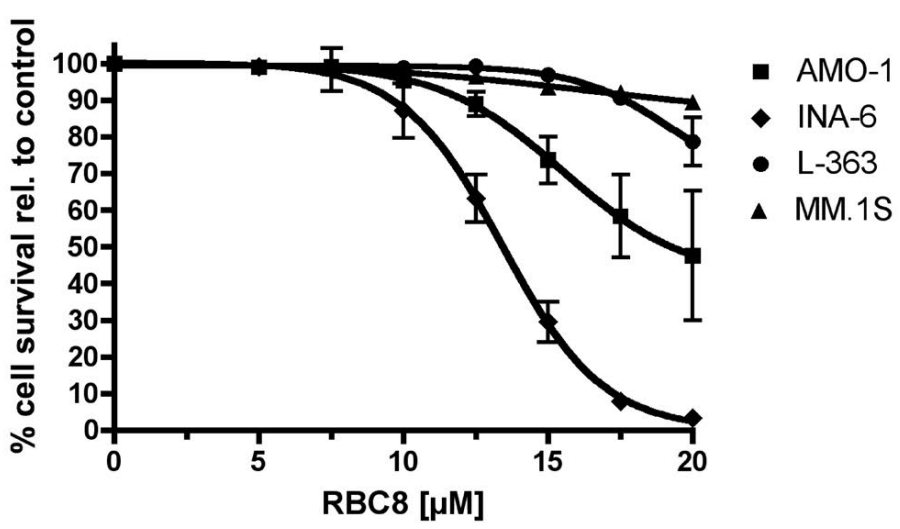

B

INA-6

MM.1S

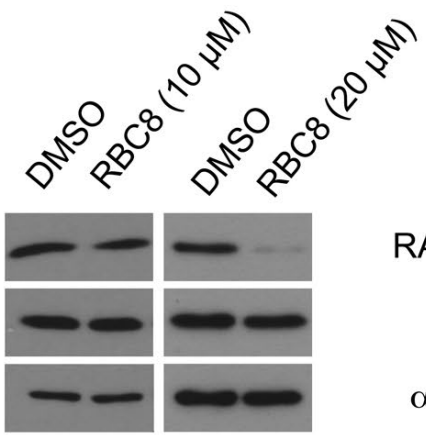

RALA-GTP pulldown

RALA total load

$\alpha$-tubulin total load

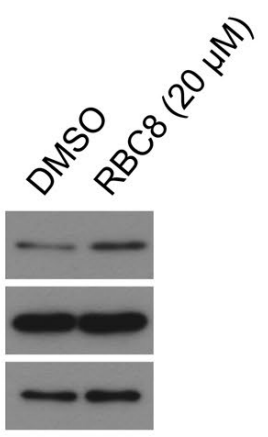

C

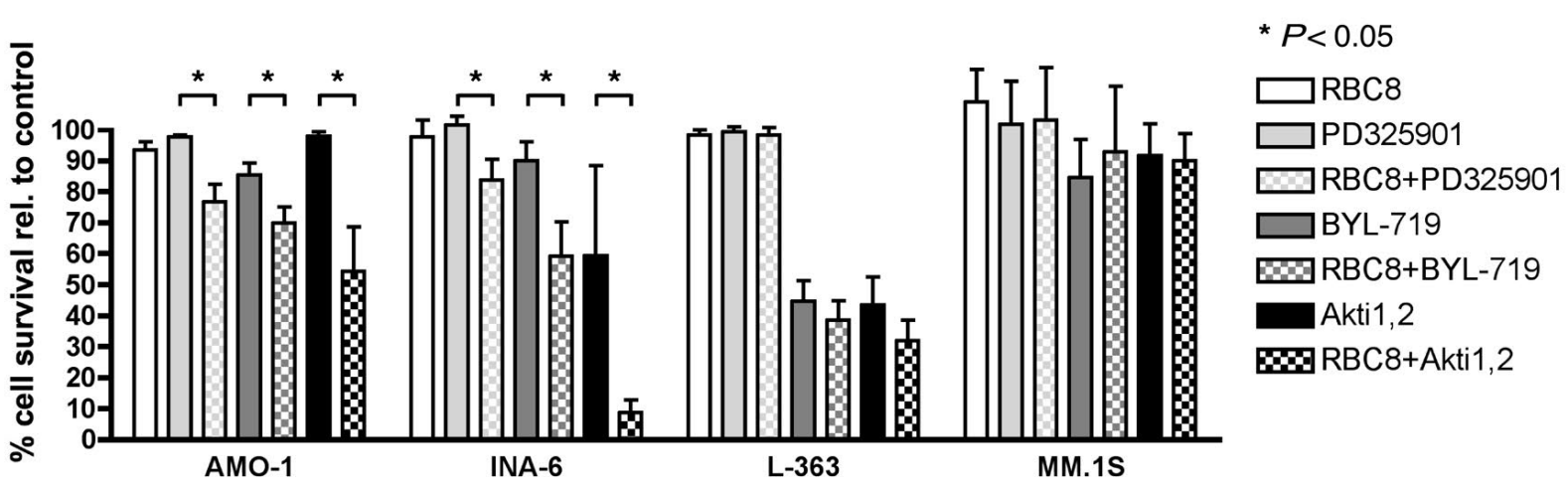

Figure 4. Pharmacological RAL inhibition in multiple myeloma cell lines. (A) AMO-1, INA-6, L-363 and MM.1S cells were subjected to increasing concentrations of the RAL inhibitor RBC8. Cell survival was measured by annexin V/PI staining after 72 hours (h) of treatment. RBC8 treatment reduced cell survival of AMO-1 and INA-6 at concentrations higher $10 \mu \mathrm{M}$. In contrast, L-363 and MM.1S cells showed no sensitivity towards RBC8 treatment with concentrations up to $20 \mu \mathrm{M}$. (B) Effect of RBC8 treatment on RAL activation status was tested in INA- 6 and MM.1S cells. INA-6 cells were treated with $10 \mu M$ and $20 \mu M$ of RBC 8 for 3 h, MM.1S cell were treated with $20 \mu \mathrm{M}$ of RBC8. RAL activation assays were performed subsequently. RALA total load served as loading control in addition to $\alpha$-tubulin. RAL-GTP levels were not influenced by treatment with RBC8 in MM.1S. In INA-6 $20 \mu \mathrm{M}$ of RBC8 reduced the amount of RAL-GTP compared to DMSO-treated cells, while RAL-GTP levels of cells treated with $10 \mu \mathrm{M}$ of RBC remained unchanged. (C) Combined blockade of RAL and PI3K/Akt or MEK/MAPK signaling. MM cell lines ( $\mathrm{n}=4$ ) were treated for $72 \mathrm{~h}$ with $10 \mu \mathrm{M}$ of RBC8, $1 \mu \mathrm{M}$ of PD0325901, $10 \mu \mathrm{M}$ (AMO-1, INA-6, L-363) or $2.5 \mu \mathrm{M}$ (MM.1S) of BYL-719, 10 $\mu \mathrm{M}$ (AMO-1, L-363) or $5 \mu \mathrm{M}$ (INA-6, MM.1S) of Akti-1,2 and the combination of RBC8 with one of the other drugs. In AMO-1 and INA-6 combination of RAL-blockade by RBC8 with blockade of MEK, PI3K or Akt1,2 by PD0325901, BYL-719 or Akti-1,2, respectively, led to a significant reduction in cell survival. MM.1S and L-363 showed no stronger decrease in cell survival after combination of RAL blockade with MEK, PI3K or Akt. Cell viability was measured with annexin V/PI staining. Bar charts show mean values and standard deviation (SD) from three independent experiments. Percentages were calculated relative to DMSO-treated control. 

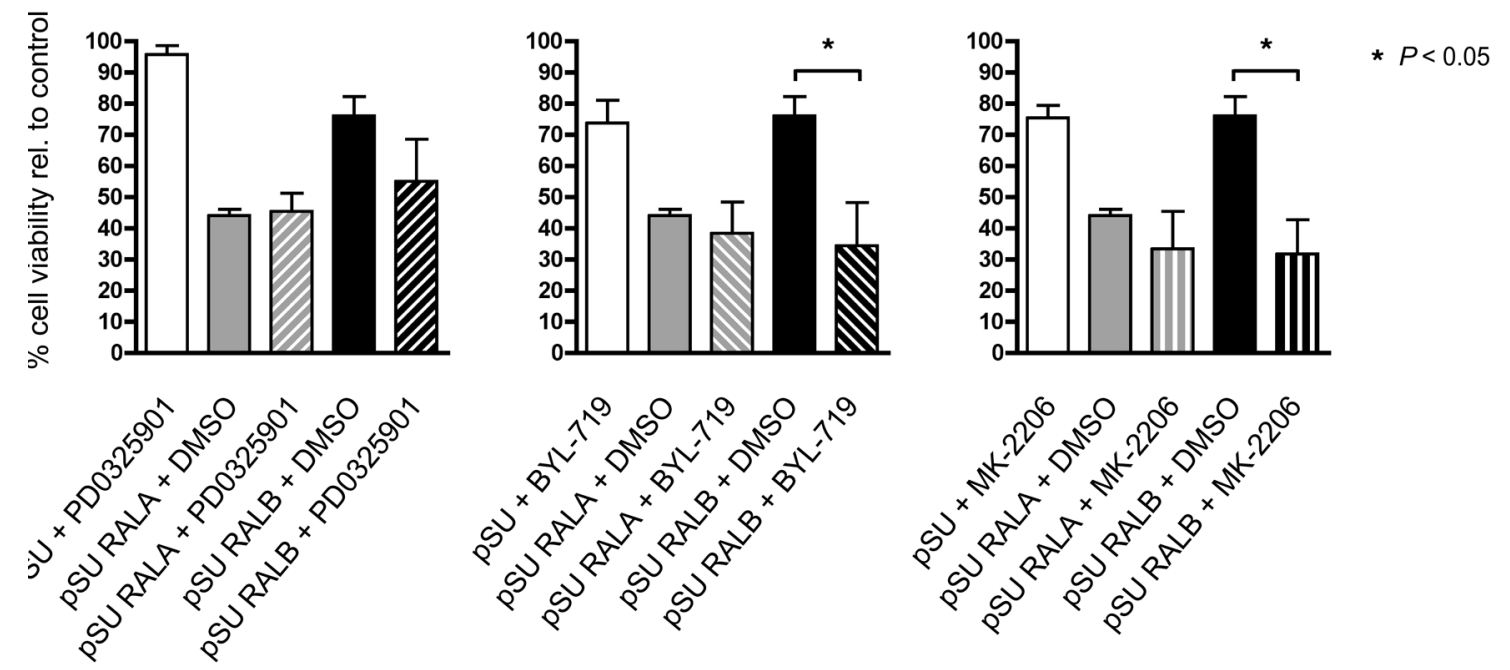

Figure 5. Combined RAL-knockdown and blockade of PI3K/Akt or MEK/MAPK signaling. L-363 cells were transfected with shRNA-expression vectors against RALA or RALB, purified next day by selection for strongly transfected cells, and then treated with PD0325901 (1 $\mu$ M), MK2206 (1 $\mu \mathrm{M})$ or BYL-719 (2.5 $\mu \mathrm{M})$. Cell survival was measured by annexin V/PI staining after 2 days (= day 3 post-transfection). Combination of RALA knockdown with PD0325901, BYL-719 or MK-2206 treatment did not further enhance the already strong apoptosis-induction resulting from RALA depletion alone. RALB knockdown in combination with PD0325901 showed only slight additional apoptosis-induction, whereas in combination with BYL-719 or MK-2206 treatment, the rate of cell death was strongly enhanced and matched that achieved by RALA knockdown.

\section{RNAi-mediated RAL knockdown induces cell death in MM cells}

To assess whether RAL proteins contribute to MM cell survival we used an RNAi-mediated knockdown approach in HMCL with subsequent cell death assays and Western analysis. Two different target sequences against each of the respective isoforms, RALA and RALB, were cloned into pSUPER-type shRNA expression vectors. Cell survival was quantified 3 and 4 days after transfection by flow cytometry and assessment of annexin V-FITC-negative/ PI-negative events. In 4 of 7 cell lines tested, and with both shRNA-constructs, RALA depletion yielded stronger cell death effects than knockdown of RALB (Figure 2A-B and Online Supplementary Figure S2). Specifically, for cell line L363 , viability decreased to below $40 \%$ (day 3 post-transfection) and to below $30 \%$ (day 4 post-transfection) of control cells after RALA knockdown (Figure 2A). Knockdown of RALB, too, led to significantly decreased viability, albeit to a lesser extent $(63-71 \%$ at day 3 post-transfection, $39-59 \%$ at day 4 post-transfection (Figure 2A).

Similarly, in MM.1S cells, knockdown of RALA led to significantly reduced cell survival to $57-64 \%$ at day 3 and to $32-42 \%$ at day 4 post-transfection. Knockdown of RALB significantly induced apoptosis leading to cell survival rates of $69-87 \%$ at 3 days and $52-79 \%$ at 4 days after electroporation (Figure 2B).

RAL knockdown also led to cell death in other MM cell lines tested (INA-6, KMS-11, KMS-12-BM, and U-266), whereas AMO-1 cells remained largely unaffected by RAL depletion (Online Supplementary Figure S2 and Online Supplementary Table S3). Of note, concomitant knockdown of both RALA and RALB (tested in cell lines MM.1S, L-363, and INA-6) resulted in rapid and near complete cell death, precluding further functional analyses (data not shown).

Effects on cell metabolism and cell cycle distribution were less pronounced than the induction of apoptosis described above. The Alamar Blue mitochondrial activity assay showed a significant decrease to $64 \%$ in L-363 cells after RALA knockdown, but only minor effects were found for MM.1S cells (Online Supplementary Figure S3A). Likewise, RALA knockdown in L-363 cells led to a significant increase of the G2/M-phase from $16 \%$ to $27 \%$ after 2 days at the expense of the S-phase (decreased from $36 \%$ to $21 \%$ ). After 3 days, similar effects were observed for both RALA and RALB knockdown (G2/M-phase: 20\% > $24 \%$ or $25 \%$, respectively; S-phase: $34 \%>24 \%$ or $22 \%$, respectively). For MM.1S cells, the most notable change occurred after 3 days, at which time point the share of cells in S-phase had decreased from $20 \%$ to $14 \%$ after RALA knockdown, and to 10\% after RALB knockdown (Online Supplementary Figure S3B).

\section{Targeting of RAL does not affect activity of the MEK/MAPK pathway but RALA appears to sustain AKT activity}

To investigate whether cell death induction after RAL knockdown is linked to down-regulation of the classical RAS downstream apoptosis and proliferation pathways, we analyzed the phosphorylation levels of ERK1/2 (MEK/MAPK pathway) and of Akt and GSK-3 (PI3K/Akt pathway) in L-363 and MM.1S cells after knockdown of either RALA or RALB by Western blotting. Cells were harvested at day 2 after transfection, i.e. before the onset of significant amounts of cell death, and at day 3 , at which time-point care was taken to perform sample collection such that equivalent numbers of trypan-blue negative cells were collected for control and RAL-knockdown samples. RALA or RALB depletion had no discernible effect on the phosphorylation levels of any of the above-mentioned signaling intermediates at day 2 (Figures 2C-D), whereas RALA knockdown specifically led to lower levels of phos- 
pho-Akt (of both, the Thr308 and Ser473 phosphorylation sites) at day 3 (Figures $2 \mathrm{E}-\mathrm{F}$ ). This effect was quite pronounced in both of these cell lines which display relatively high constitutive levels of activated Akt.

\section{Constitutive RAL activation in MM cells remains unaffected by knockdown of oncogenic RAS}

To address our hypothesis that RAL activation is dependent on oncogenic RAS, we analyzed the change of the levels of activated GTP-bound RAL after knockdown of oncogenic KRAS or NRAS in HMCL harboring the respective mutated RAS isoform. Effective silencing of oncogenic RAS was verified by Western analysis 48 hours (h) after electroporation with the respective shRNA expression vectors. RAL-GTP levels were measured by performing RALA pulldown using RALBP1 protein-binding domain agarose beads. Notably, the RAL activation status was not altered by knockdown of oncogenic KRAS in MM.1S cells or oncogenic NRAS in INA-6 cells (Figure 3A). Expression levels of total load of RALA proteins remained also unchanged.

\section{RNAi-mediated knockdown of KRAS or RALA in MM.1S cells entails distinct transcriptomic effects}

Because mutated RAS did not appear to be directly linked to RAL activation, we next analyzed in more detail the influence of both signaling hubs on the transcriptome of KRAS-mutated MM.1S cells using an RNA sequencing technique for 28,440 gene transcripts. Cells were transfected with shRNA expression vectors against either KRAS or RALA, or with the pSU empty-vector. Strongly transfected cells were purified via CD $4 \Delta$ microbead selection and harvested at day 2 post-transfection for preparation of samples for transcriptomic analysis and Western blotting to confirm successful target knockdown (Figure 3B). As displayed in the Venn diagram (Figure 3C), KRAS knockdown led to changes in gene transcription in about double the number of genes $(n=1,473)$ than RALA knockdown $(n=771)$. Taken together, the number of transcripts that is altered in a mutually exclusive fashion $(n=1,744)$ far outweighs the number affected by both, KRAS- or RALAknockdown $(n=235)$. Using the Molecular Signatures Database Hallmark Gene Set Collection, ${ }^{40}$ ontology gene mapping was performed for the classification of differential gene expression after RAL versus RAS knockdown, highlighting the most distinct functional gene groups with relevance for MM biology (Figure 3D).

\section{Effects of the small molecule compound RBC8 on survival and RAL activation of MM cells}

The small molecule inhibitor RBC8 has recently been described as selective allosteric inhibitor of RALA and RALB, which stabilizes RAL in its inactive GDP-bound state. ${ }^{41}$ We treated MM cell lines $(n=4)$ and primary MM cells $(n=6)$ for 3 days with different concentrations of RBC8 and measured cell survival by flow cytometry using annexin V/PI staining. INA-6 cells were most sensitive to the drug with EC50/90 values of 12,5 and $17,5 \mu \mathrm{M}$, respectively. MM.1S cells, on the other hand, were unaffected by RCB-8 even at the highest concentration tested $(20 \mu \mathrm{M})$ (Figure 4A). In accordance with these results, analysis of RALA activation by pulldown of RALA-GTP in the sensitive INA- 6 cells showed a strong reduction after treatment with $20 \mu \mathrm{M}$ of RBC8 for $3 \mathrm{~h}$, whereas at $10 \mu \mathrm{M}$ no marked effects were seen. Conversely, the levels of activated
RALA remained unaltered after $3 \mathrm{~h}$-treatment with $20 \mu \mathrm{M}$ of RBC8 in MM.1S cells (Figure 4B). Primary MM samples remained largely unaffected by RBC8 $(20 \mu \mathrm{M})$ (Online Supplementary Figure S5A).

Data from combined RAL (RBC8) and MEK/MAPK or PI3K/Akt inhibition showed increased anti-myeloma effects in RBC8-sensitive cell lines, but no combination advantage in RBC8-insensitive cells (Figure 4C). These drug combinations showed at best mild effects on cell survival in the primary MM samples tested (Online Supplementary Figure S5B).

We also performed Alamar Blue assays to test possible effects of RBC8 treatment on cell metabolism and proliferation. We found only minor impacts at concentrations up to $20 \mu \mathrm{M}$, most pronounced in INA-6 and AMO1 cells (Online Supplementary Figure S3C). Although higher concentrations (up to $40 \mu \mathrm{M}$ ) of RBC8 enhanced these effects, these concentrations may also exert unspecific cytotoxicity. ${ }^{42}$ Cell cycle distribution after RBC8 treatment was analyzed by BrdU/PI staining and revealed that in INA-6 cells which are most sensitive to treatment with RBC8, the G2/ M-phase significantly increased from 19\% to $30 \%$ at the expense of the S-phase, which decreased from 38\% to 20\% (Online Supplementary Figure S3D). No relevant changes were observed in AMO-1, L-363, or MM.1S cells. Finally, no effects on the constitutive levels of phospho-ERK and phospho-Akt were observed after RBC8 treatment of HCML (Online Supplementary Figure S4).

\section{Combination of RALB depletion with PI3K/Akt inhibitors leads to enhanced MM cell death}

Because RAL blockade had either no or differential effects on the activity of the MEK/MAPK or PI3K/Akt pathways (see above and Figures 2C-F), we tested the potential usefulness of pharmacologically targeting these pathways in combination with RAL knockdown.

After knockdown of either RALA or RALB, L-363 cells were treated with pharmacological inhibitors of MEK1/2 (PD0325901), Akt (MK2206) or PIK3CA (BYL-719). While combinations of either of these compounds with RALA abrogation did not significantly enhance apoptosis induction in excess of the rather strong effects of RALA knockdown alone, combined depletion of RALB and Akt or PI3K inhibition, respectively, led to significantly higher rates of cell death (Figure 5).

Combination experiments in $\mathrm{MM}$ cell lines using the pharmacological RAL inhibitor RBC8 showed statistically significant (and functionally relevant) synergistic effects for the combination with PI3K/Akt primarily in the aforementioned cell line INA-6 (Figure 4C).

\section{Mass spectrometric analysis identifies the exocyst complex as a predominant RAL interaction partner}

To analyze potential downstream signaling partners of RAL in MM cells, we performed quantitative mass spectrometry of MM.1S cells with stable expression of HAtagged RALA protein. A total of 48 proteins were identified as specific partners of RALA, including six members of the exocyst complex (EXOC-1, 2, 3, 4, 7, 8) (Online Supplementary Figure S6). Except for EXOC-7, all of these exocyst components are listed as highly confident interaction partners in the HitPredict database for protein-protein interactions. ${ }^{43}$ Moreover, EXOC-2/Sec5 and EXOC8/Exo84 have previously been described to play a role in RAL-mediated tumor cell proliferation. ${ }^{44}$ 


\section{Discussion}

In this study, we demonstrate the functional importance of the small GTPase RAL for survival of MM cells. Both RAL isoforms were strongly expressed in the majority of HMCL and primary MM cells when directly compared to normal plasma cells or pre-malignant MGUS cells. Moreover, GTP-bound and thus activated RAL was present in all MM cell lines analyzed, pointing to a potential functional role of RAL in transition to and/ or maintenance of the malignant tumor clone. To test this hypothesis we performed isoform-specific RNAi-mediated RAL knockdown and found that abrogation of RAL led to fast and strong cell death induction in the majority of MM cell lines. These experiments thus identified the RAL GTPases as potent pro-survival mediators in MM.

Because activation of RAL has been described as a predominantly RAS-dependent oncogenic survival pathway in various cancer entities, ${ }^{29,45-48}$ we also sought to test the putative functional link between oncogenic RAS and RAL activation in MM using pulldown assays and RNA sequencing. We found that in the MM cells tested, RAL activation could not be ascribed to the presence of oncogenic RAS (as defined by harboring activating point mutations in NRAS or KRAS). Neither did shRNA-mediated knockdown of oncogenic RAS alter the RAL activation status. As opposed to the well-defined activating mutations of NRAS and KRAS, data available from the CoMMpass trial cohort (these data were generated as part of the Multiple Myeloma Research Foundation Personalized Medicine Initiatives [https://research.themmrforg and www.themmrf.org]) and other large next generation sequencing studies ${ }^{49-51}$ revealed no oncogenic bona fide mutations affecting RAL in MM. Activation of RAL by RAS-independent mechanisms has also been shown by other groups in solid tumors such as melanoma, ${ }^{52}$ bladder carcinoma ${ }^{53}$ and malignant peripheral nerve sheath tumor ${ }^{54}$ in which deregulated RAL guanine exchange factors, direct RAL phosphorylation by protein kinase C, or involvement of secondary GTPases, respectively, may lead to increased RAL-dependent tumor cell proliferation.

De Gorter et al. showed that RAL could be activated by chemokines originating from the bone marrow microenvironment. In their study, treatment of MM cells with stromal cell-derived factor-1 resulted in increased levels of GTP-bound RAL and led to enhanced cell migration..$^{55}$ These effects occurred independently of RAS, which is in line with our observation that no direct link between oncogenic RAS and activated RAL could be established.

Additionally, in our transcriptome analysis we observed distinct changes of gene expression after RAL versus RAS knockdown, underpinning the notion that RAL functions as a survival pathway in its own right and warrants further validation for potential therapeutic intervention.

Due to the high affinity of the guanine nucleotides at their binding sites, small GTPases such as RAS and RAL are hard to target pharmacologically, however. Whereas to date, no clinically suitable RAS inhibitors are available, $e^{1,456}$ a small molecule RAL inhibitor has recently been described, ${ }^{41}$ showing in vitro effects in adipose tissue ${ }^{57}$ and in chronic myelogenous leukemia. ${ }^{58}$ This allosteric compound has been developed to stabilize RAL in its inactive GDP-bound state and thus prevent its activation. ${ }^{41}$ In our hands, in the most sensitive MM cell line INA-6, RAL acti- vation was indeed abrogated and apoptosis induced at drug concentrations starting from $10 \mu \mathrm{M}$, whereas survival of primary MM cells and of other HMCL was less affected even at $20 \mu \mathrm{M}$, warranting development of more potent second generation RAL inhibitors. To this end, Walsh et al. ${ }^{42}$ have recently observed in a murine platelet RAL knockout model that RBC8 does indeed exert specific as well as unspecific effects within similar concentration ranges, which may explain its inconsistency when tested across different (cell line) models.

In our mass-spectrometric analysis which we performed to define RAL interaction partners serving as potential downstream mediators of the RAL pathway, we identified six members of the exocyst complex among the highest scoring hits. They included the complex members EXOC2/Sec5 and EXOC-8/Exo84 which are known to contribute to RAL-induced proliferation in tumor cells. ${ }^{4}$ Interestingly, RALBP1 which is another well-defined binding partner to RAL, did not appear to play a predominant role in our MM cell line analysis.

In $\mathrm{MM}$, the interconnection with signals from a per se altered bone marrow microenvironment ${ }^{59-61}$ may bypass otherwise important signaling hubs such as RAS. We have previously made this observation for the PI3K/Akt pathway in MM, which can be constitutively activated independently of oncogenic RAS, possibly by involvement of upstream receptor tyrosine kinases. ${ }^{12,13,51}$ Whereas we found no indication for RAL involvement in RAS/MAPK signaling, we did find distinctly lower levels of activated Akt after extended knockdown of RALA. In keeping with this observation, while the already strong apoptotic effects of RALA depletion could not further be enhanced by simultaneous pharmacological PI3K/Akt blockade, such treatment considerably enhanced the cytotoxic effects of RALB knockdown. These observations suggest that both RAL isoforms may at least in parts play differential roles in cellular signaling, and point specifically to a role for RALA as one of the potential mediators for high intrinsic levels of active Akt in a subgroup of MM cells.

Given the heterogeneity of oncogenic pathways in MM, synergistically acting combination therapies seem to be the most promising targeted treatment strategies. To this extent, our data demonstrate that RAL abrogation may be effective in combination with inhibitors of the PI3K/Akt pathway. This is particularly important because in early clinical trials, PI3K inhibitors displayed limited effectivity and will therefore most likely play a role as combination partners in tumor therapy. ${ }^{18,62}$

Taken together, our data indicate that RAL is a promising molecular target for MM therapy that is functionally independent of oncogenic RAS. However, because the one existing pharmacological inhibitor targeting RAL in our hands does not perfectly mimmick the strong effects of RAL knockdown, development of more potent secondgeneration inhibitors for $\mathrm{MM}$ treatment is mandatory for clinical translation.

\section{Funding}

This work was supported by grants from the Deutsche Forschungsgemeinschaft (KFO 216) and the Interdisziplinäres Zentrum für Klinische Forschung of the Universitätsklinikum Würzburg (B-188). TSte was supported by a fellowship of the Else Kröner Fresenius-Stiftung (2010_Kolleg.52). EL and RB were supported by a grant of the Deutsche Krebshilfe (70112693). 


\section{References}

1. Cox AD, Fesik SW, Kimmelman AC, Luo J, Der CJ. Drugging the undruggable RAS: Mission Possible? Nat Rev Drug Discov. 2014;13(11):828-851

2. Stephen AG, Esposito D, Bagni RK, McCormick F. Dragging Ras back in the ring. Cancer Cell. 2014;25(3):272-281.

3. Athuluri-Divakar SK, Vasquez-Del Carpio $\mathrm{R}$, Dutta K, et al. A small molecule RASmimetic disrupts RAS association with effector proteins to block signaling. Cell. 2016;165(3):643-655

4. McCormick F. K-Ras protein as a drug target. J Mol Med. 2016:94(3):253-258.

5. Beeram M, Patnaik A, Rowinsky EK. Raf: A strategic target for therapeutic development against cancer. J Clin Oncol. 2005; 23(27):6771-6790

6. Baines AT, Xu D, Der CJ. Inhibition of Ras for cancer treatment: the search continues. Future Med Chem. 2011;3(14):1787-1808.

7. Mandal R, Becker S, Strebhardt K. Stamping out RAF and MEK1/2 to inhibit the ERK1/2 pathway: an emerging threat to anticancer therapy. Oncogene. 2016; 35(20):2547-2561.

8. Steinbrunn T, Stühmer T, Gattenlöhner S, et al. Mutated RAS and constitutively activated Akt delineate distinct oncogenic pathways, which independently contribute to multiple myeloma cell survival. Blood. 2011:117(6):1998-2004.

9. Lionetti $M$, Barbieri $M$, Todoerti $\mathrm{K}$, et al. Molecular spectrum of BRAF, NRAS and KRAS gene mutations in plasma cell dyscrasias: implication for MEK-ERK pathway activation. Oncotarget. 2015; 6(27):24205-24217

10. Walker BA, Boyle EM, Wardell CP, et al. Mutational spectrum, copy number changes, and outcome: results of a sequencing study of patients with newly diagnosed myeloma. J Clin Oncol. 2015;33(33):39113920.

11. Steinbrunn T, Stühmer T, Sayehli C, et al. Combined targeting of MEK/MAPK and PI3K/Akt signalling in multiple myeloma. Br J Haematol. 2012;159(4):430-440.

12. Hofmann C, Stühmer T, Schmiedl N, et al. PI3K-dependent multiple myeloma cell survival is mediated by the PIK3CA isoform. Br J Haematol. 2014;166(4):529-539.

13. Munugalavadla V, Mariathasan S, Slaga D, et al. The PI3K inhibitor GDC-0941 combines with existing clinical regimens for superior activity in multiple myeloma. Oncogene. 2014;33(3):316-325.

14. Müller E, Bauer S, Stühmer T, et al. Pan-Raf co-operates with PI3K-dependent signalling and critically contributes to myeloma cell survival independently of mutated RAS. Leukemia. 2017;31(4):922-933.

15. Xu J, Pfarr N, Endris V, et al. Molecular signaling in multiple myeloma: association of RAS/RAF mutations and MEK/ERK pathway activation. Oncogenesis. 2017; 6(5):e337.

16. McMillin DW, Ooi M, Delmore J, et al. Antimyeloma activity of the orally bioavailable dual phosphatidylinositol 3kinase/mammalian target of rapamycin inhibitor NVP-BEZ235. Cancer Res. 2009; 69(14):5835-5842.

17. Mayer IA, Arteaga CL. The PI3K/AKT pathway as a target for cancer treatment. Annu Rev Med. 2016;67(1):11-28.

18. Ramakrishnan V, Kumar S. $\mathrm{PI} 3 \mathrm{~K} / \mathrm{AKT} / \mathrm{mTOR}$ pathway in multiple myeloma: from basic biology to clinical promise. Leuk Lymphoma. 2018; 59(11):2524-2334.

19. Breitkreutz I, Podar K, Figueroa-Vazquez V et al. The orally available multikinase inhibitor regorafenib (BAY 73-4506) in multiple myeloma. Ann Hematol. 2018; 97(5):839-849.

20. Chang-Yew Leow C, Gerondakis S, Spencer A. MEK inhibitors as a chemotherapeutic intervention in multiple myeloma. Blood Cancer J. 2013;3(3):e105.

21. Holkova B, Zingone A, Kmieciak M, et al A phase II trial of AZD6244 (Selumetinib, ARRY-142886), an oral MEK1/2 inhibitor, in relapsed/refractory multiple myeloma. Clin Cancer Res. 2016;22(5):1067-1075.

22. Ramakrishnan V, D'Souza A. Signaling pathways and emerging therapies in multiple myeloma. Curr Hematol Malig Rep. 2016;11(2):156-164.

23. Bedard PL, Tabernero J, Janku F, et al. A phase Ib dose-escalation study of the oral Pan-PI3K inhibitor buparlisib (BKM120) in combination with the oral MEK1/2 inhibitor trametinib (GSK1120212) in patients with selected advanced solid tumors. Clin Cancer Res. 2015;21(4):730738.

24. Tolcher AW, Patnaik A, Papadopoulos KP, et al. Phase I study of the MEK inhibitor trametinib in combination with the AKT inhibitor afuresertib in patients with solid tumors and multiple myeloma. Cancer Chemother Pharmacol. 2015;75(1):183 189.

25. Grilley-Olson JE, Bedard PL, Fasolo A, et al. A phase Ib dose-escalation study of the MEK inhibitor trametinib in combination with the PI3K/mTOR inhibitor GSK2126458 in patients with advanced solid tumors. Invest New Drugs. 2016; 34(6):740-749.

26. Wainberg ZA, Alsina M, Soares HP, et al. A multi-arm phase I study of the PI3K/mTOR inhibitors PF-04691502 and gedatolisib (PF05212384) plus irinotecan or the MEK inhibitor PD-0325901 in advanced cancer. Target Oncol. 2017;12(6):775-785.

27. Rodriguez-Viciana P, McCormick F. RalGDS comes of age. Cancer Cell. 2005; $7(3): 205-206$

28. Thomas JC, Cooper JM, Clayton NS, et al Inhibition of Ral GTPases using a stapled peptide approach. J Biol Chem. 2016; 291(35):18310-18325

29. Yan C, Theodorescu D. RAL GTPases: biology and potential as therapeutic targets in cancer. Pharmacol Rev. 2018;70(1):1-11.

30. Rangarajan A, Hong SJ, Gifford A Weinberg RA. Species- and cell type-specific requirements for cellular transformation. Cancer Cell. 2004:6(2):171-183.

31. Lim K-H, O'Hayer K, Adam SJ, et al. Divergent roles for RalA and RalB in malignant growth of human pancreatic carcinoma cells. Curr Biol. 2006;16(24): 2385-2394.

32. Martin TD, Der CJ. Differential involvement of RalA and RalB in colorectal cancer. Small GTPases. 2012;3(2):126-130.

33. Stühmer T, Arts J, Chatterjee $M$, et al. Preclinical anti-myeloma activity of the novel HDAC-inhibitor JNJ-26481585. Br J Haematol. 2010;149(4):529-536.

34. Steinbrunn T, Chatterjee M, Bargou RC, Stühmer T. Efficient transient transfection of human multiple myeloma cells by eectroporation - an appraisal. PLoS One. 2014 9(6):e97443.

35. Brummelkamp TR, Bernards R, Agami R. A system for stable expression of short inter- fering RNAs in mammalian cells. Science. 2002;296(5567):550-553.

36. Lim K-H, Baines AT, Fiordalisi JJ, et al Activation of RalA is critical for Rasinduced tumorigenesis of human cells. Cancer Cell. 2005;7(6):533-545

37. Oxford G, Owens CR, Titus BJ, et al. RalA and RalB: rntagonistic Relatives in cancer cell migration. Cancer Res. 2005; 65(16):7111-7120

38. Cox J, Hein MY, Luber CA, et al. Accurate Proteome-wide Label-free Quantification by Delayed Normalization and Maximal Peptide Ratio Extraction, Termed MaxLFO. Mol Cell Proteomics. 2014;13(9):2513 2526.

39. Cox J, Mann M. MaxQuant enables high peptide identification rates, individualized p.p.b.-range mass accuracies and proteome-wide protein quantification. Nat Biotechnol. 2008;26(12):1367-1372.

40. Liberzon A, Birger C, Thorvaldsdóttir $\mathrm{H}$, et al. The molecular signatures database hallmark gene set collection. Cell Syst. 2015; 1(6):417-425.

41. Yan C, Liu D, Li L, et al. Discovery and characterization of small molecules that target the GTPase Ral. Nature. 2014;515(7527):443-447.

42. Walsh TG, Wersäll A, Poole AW Characterisation of the Ral GTPase inhibitor RBC8 in human and mouse platelets. Cell Signal. 2019;59:34-40.

43. López Y, Nakai K, Patil A. HitPredict version 4: comprehensive reliability scoring of physical protein-protein interactions from more than 100 species. Database (Oxford) 2015;2015.

44. Shin H, Kaplan REW, Duong T, Fakieh R Reiner DJ. Ral signals through a MAP4 kinase-p38 MAP kinase cascade in C. elegans cell fate patterning. Cell Rep. 2018; 24(10):2669-2681.

45. González-García A, Pritchard CA, Paterson HF, et al. RalGDS is required for tumor formation in a model of skin carcinogenesis Cancer Cell. 2005;7(3):219-226.

46. Mishra PJ, Ha L, Rieker J, et al. Dissection of RAS downstream pathways in melanomagenesis: a role for Ral in transformation. Oncogene. 2010;29(16):2449-2456.

47. Yin J, Pollock C, Tracy K, et al. Activation of the RalGEF/Ral pathway promotes prostate cancer metastasis to bone. Mol Cell Biol. 2007;27(21):7538-7550.

48. Guin S, Theodorescu D. The RAS-RAL axis in cancer: evidence for mutation-specific selectivity in non-small cell lung cancer Acta Pharmacol Sin. 2015;36(3):291-297.

49. Chapman MA, Lawrence MS, Keats JJ, et al. Initial genome sequencing and analysis of multiple myeloma. Nature. 2011; 471(7339):467-472

50. Lohr JG, Stojanov P, Carter SL, et al Widespread genetic heterogeneity in multiple myeloma: implications for targeted therapy. Cancer Cell. 2014;25(1):91-101.

51. Leich E, Weißbach S, Klein H-U, et al Multiple myeloma is affected by multiple and heterogeneous somatic mutations in adhesion- and receptor tyrosine kinase signaling molecules. Blood Cancer J. 2013; 3(2):e102-e102.

52. Zipfel PA, Brady DC, Kashatus DF, et al. Ral activation promotes melanomagenesis Oncogene. 2010;29(34):4859-4864.

53. Wang $\mathrm{H}$, Owens $\mathrm{C}$, Chandra $\mathrm{N}$, et al Phosphorylation of RalB is important for bladder cancer cell growth and metastasis. Cancer Res. 2010;70(21):8760-8769.

54. Bodempudi V, Yamoutpoor F, Pan W, et al. 
Ral overactivation in malignant peripheral nerve sheath tumors. Mol Cell Biol. 2009; 29(14):3964-3974.

55. De Gorter DJJ, Reijmers RM, Beuling EA, et al. The small GTPase Ral mediates SDF-1 induced migration of $\mathrm{B}$ cells and multiple myeloma cells. Blood. 2008;111(7):33643372 .

56. Wilson CY, Tolias P. Recent advances in cancer drug discovery targeting RAS. Drug Discov Today. 2016;21(12):1915-1919.

57. Skorobogatko Y, Dragan M, Cordon C, et al. RalA controls glucose homeostasis by regulating glucose uptake in brown fat Proc Natl Acad Sci. 2018;115(30):7819. 7824

58. Gu C, Feng M, Yin Z, Luo X, Yang J. RalA, a GTPase targeted by miR 181a, promotes transformation and progression by activating the Ras related signaling pathway in chronic myelogenous leukemia. Oncotarget. 2016;7(15):20561-20573.

59. Schinke C, Qu P, Mehdi SJ, et al. The pattern of mesenchymal stem cell expression is an independent marker of outcome in multiple myeloma. Clin Cancer Res. 2018;
24(12):2913-2919

60. Garayoa M, Garcia JL, Santamaria C, et al. Mesenchymal stem cells from multiple myeloma patients display distinct genomic profile as compared with those from normal donors. Leukemia. 2009;23(8):1515 1527

61. Bianchi G, Munshi NC. Pathogenesis beyond the cancer clone(s) in multiple myeloma. Blood. 2015;125(20):3049-3058.

62. Markman B, J. Tao J, Scaltriti M. PI3K pathway inhibitors: better not left alone. Curr Pharm Des. 2013;19(5):895-906. 